\title{
CORRELAÇÕES FENOTÍPICAS ENTRE TAMANHO DE GRÃOS E OUTROS CARACTERES EM TOPOCRUZAMENTOS DE SOJA TIPO ALIMENTO COM TIPO GRÃO ${ }^{1}$
}

\author{
GILBERTO KEN-ITI YOKOMIZO², JOÃO BATISTA DUARTE³ e NATAL ANTONIO VELLO ${ }^{4}$
}

\begin{abstract}
RESUMO - Este trabalho foi conduzido para avaliar a correlação fenotípica entre vários caracteres em topocruzamentos entre soja tipo alimento e soja tipo grão (Doko e FT-2). Valores de interesse para facilitar a seleção foram obtidos nas correlações entre peso de cem sementes (tamanho de sementes) e dias para atingir a maturidade, e entre tamanho de sementes e largura visual da vagem. Na correlação entre produtividade de grãos e tamanho de sementes somente os topocruzamentos com Doko foram significativos. As estimativas de correlações em plantas individuais tenderam a confirmar aquelas obtidas em médias de parcelas. Os valores de correlação foram diferentes entre os tipos (grão, broto/"natto" e hortaliça) de soja envolvidos, o que sugere a adoção de estratégias de seleção distintas. O estudo de correlações para cada topocruzamento é importante, pois podem ocorrer diferenças no desempenho das plantas como foi observado nos resultados obtidos.
\end{abstract}

Termos para indexação: Glycine max, genótipos, critérios de seleção, características agronômicas, produtividade, melhoramento de plantas.

\section{PHENOTYPIC CORRELATION BETWEEN SEED SIZE AND OTHER CHARACTERISTICS IN TOPCROSSES OF VEGETABLE SOYBEAN WITH GRAIN TYPE}

\begin{abstract}
This work was conducted to evaluate the phenotypic correlation between characters in topcrosses between vegetable soybean with grain type cultivars (Doko and FT-2). Interesting values to facilitate selections were observed in correlations between one hundred seed weight (seed size) and days of maturation and between seed size and pod width as visual score. Seed yield and seed size were significantly correlated only in Doko's topcrosses. Estimated correlation from individual plants tended to confirm those obtained at mean level plot. Depending on the soybean type (grain, sprout/natto and vegetable), the correlation values were different. Because of that it is recommended different selection strategies to evaluate distinct soybean classes. Correlation studies for each topcross are important, due to differences in plant performance as observed in obtained results.
\end{abstract}

Index terms: Glycine max, genotypes, selection criteria, agronomic characters, productivity, plant breeding.

${ }^{1}$ Aceito para publicação em 21 de janeiro de 2000.

${ }^{2}$ Eng. Agrôn., Dr., Embrapa-Centro de Pesquisa Agroflorestal do Amapá (CPAF-Amapá), Rod. JK, km 5, Caixa Postal 10, CEP 68902-280 Macapá, AP. E-mail: gilberto@cpafap.embrapa.br

${ }^{3}$ Eng. Agrôn., Prof. Assistente, Escola de Agronomia, Universidade Federal de Goiás, Caixa Postal 131, CEP 74001-970 Goiânia, GO. E-mail: jduarte@uol.com.br

${ }^{4}$ Eng. Agrôn., Prof. Titular, Dep. de Genética, Escola Superior de Agricultura Luiz de Queiroz (ESALQ), Caixa Postal 83, CEP13400-970 Piracicaba, SP.E-mail: naavello@ carpa.ciagri.usp.br

\section{INTRODUÇÃO}

A soja é de grande importância econômica para o Brasil, sendo a segunda cultura em produção de grãos, superada apenas pelo milho. Seu consumo, quase na totalidade, está na forma de óleo e farelo, este último destinado à alimentação animal. No Brasil, o consumo de soja diretamente na alimentação humana é muito restrito, ao contrário do que ocorre em diver- 
sos países orientais, cujo consumo é verificado há pelo menos três milênios. Existem genótipos específicos de soja em função de seu uso, deste modo a classificação da soja pode ser feita em dois grupos principais: tipo grão e tipo alimento. A soja tipo grão apresenta sementes de tamanho médio, representado pelo peso de 100 sementes (PCS) entre 10 e $19 \mathrm{~g}$, com sabor desagradável quando consumido na alimentação humana direta, sendo cultivada principalmente para atender às indústrias brasileira e estrangeira de farelo e óleo. A soja tipo alimento, com sabor agradável, apresenta duas categorias: sementes pequenas, com PCS menor que $10 \mathrm{~g}$, destinadas ao consumo em forma de brotos e de "natto" (fermentado); e sementes grandes, com PCS igual ou maior que $20 \mathrm{~g} / 100$ sementes. A soja com sementes grandes tem os seguintes usos principais: soja hortaliça (vegetable soybean, green soybean, ou edamame), utilizada para consumo humano direto na forma de vagens imaturas fase $\mathrm{R}_{6}$ (Fehr \& Caviness, 1977); soja doce (kuromame), compreendendo genótipos com sementes grandes, de tegumento preto e consumidos na forma de grãos maduros; soja salada, para preparação de saladas mistas (soja misturada com hortaliças e legumes), com sementes maduras grandes e tegumento de coloração amarela, verde ou variegada.

Sabe-se também que a soja tipo alimento, além do tamanho das sementes, apresenta características distintas da comumente encontrada no mercado brasileiro (tipo grão), tais como: cor do tegumento, hilo e sabor e odor delicados (Vello, 1992). A proteína da soja é a única do reino vegetal com possibilidade de substituir as proteínas animais, do ponto de vista nutricional, pois contém todos os aminoácidos essenciais, e em proporção adequada, excetuando-se apenas os aminoácidos sulfurados (metionina e cistina), com níveis baixos de concentração (Canto $\&$ Turatti, 1989).

Um fator de suma importância em cultivares de soja é a produtividade, além de resistência a pragas e doenças. Além disso, a soja tipo alimento também deve apresentar tamanho adequado dos grãos conforme usos específicos. Portanto, o conhecimento da grandeza da associação entre esses caracteres são primordiais num programa de melhoramento, pois permite ao melhorista saber como a seleção de uma característica pode causar alterações em outras (Johnson et al.,1955; Vencovsky, 1992). Também é possível estabelecer o melhoramento indireto de um caráter, que apresente herança complexa, baixa herdabilidade ou manifestação em gerações avançadas através da seleção em um outro caráter, com herança mais simples ou de maior herdabilidade. Porém, é importante ater-se a resultados de correlações diferentes em função do tipo de parcela empregada nos estudos, conforme pôde ser observado nos trabalhos de Moro et al. (1992), em que parcelas representadas por linhas apresentaram um determinado valor de correlação e em covas, outro.

A pesquisa teve como objetivo estimar correlações fenotípicas entre plantas individuais de soja e médias de parcelas, em relação a tamanho de sementes, número de dias para maturidade, nota de valor agronômico, produtividade de grãos e nota de largura visual das vagens de várias progênies oriundas de topocruzamentos entre genótipos tipo grão (Doko e FT-2) e genótipos exóticos tipo alimento, tanto de sementes pequenas como de sementes grandes.

\section{MATERIAL E MÉTODOS}

O experimento foi conduzido na Escola Superior de Agricultura Luiz de Queiroz/Universidade de São Paulo, em área do Departamento de Genética, Piracicaba, SP. O material genético envolvido nesta pesquisa constou de 72 topocruzamentos em geração $\mathrm{F}_{5: 4}$, sendo 67 provenientes do cruzamento de materiais exóticos tipo hortaliça com as cultivares FT-2 e Doko (30 e 37, respectivamente), e cinco de materiais exóticos do tipo broto/"natto" também com Doko e FT-2 (3 e 2, respectivamente).

A semeadura foi realizada no verão, em três datas (30 de outubro, 5 e 20 de novembro), sendo cada planta $F_{4}$ representada por uma cova com uma a cinco plantas $F_{5}$. A parcela foi composta por três fileiras com quatro covas cada, perfazendo 12 covas de um topocruzamento por repetição. O espaçamento utilizado foi de $0,5 \mathrm{~m}$ entre covas e $0,5 \mathrm{~m}$ entre fileiras. O delineamento adotado foi o de blocos casualizados, com duas repetições por época, totalizando 72 covas por topocruzamento. A disposição dos topocruzamentos dentro dos blocos foi feita por sorteio; além disso a área apresentava solo homogêneo, evitando-se o favorecimento de algum topocruzamento em relação a outro.

Os caracteres avaliados foram: número de dias para maturidade (NDM), contados da semeadura à colheita; 
valor agronômico (VA), com notas visuais de 1 (planta ruim) a 5 (planta ótima), baseada em características agronômicas da planta, como arquitetura, sanidade, produtividade, presença ou não de retenção foliar e haste verde; produtividade de grãos (PG), em gramas por cova; peso de cem sementes (PCS), obtido pela pesagem de uma amostra aleatória de cem sementes de cada cova, em gramas; e largura visual das vagens (LVV), baseada numa escala de notas visuais de 1 (vagens estreitas) a 5 (vagens largas).

A análise de variância foi realizada para cada ambiente, e após a verificação da homogeneidade dos resíduos e os devidos ajustes, foi realizada a análise de variância conjunta. Com base nesta análise, foram estimadas as correlações com o auxílio do programa computacional SAS (Statistical Analysis System) desenvolvido pelo SAS Institute Inc., USA. Foram estimados coeficientes de correlação fenotípica linear de Pearson para os valores de plantas individuais, e médias de tratamentos para os grupos formados em função do tamanho de sementes, ou seja, os grupos dos topocuzamentos de sementes grandes obtidos com Doko ou FT-2 e o grupo dos topocruzamentos de sementes pequenas, obtidos com Doko e com FT-2. Também foram estimadas correlações fenotípicas para cada topocruzamento individualmente para avaliação do valor mínimo e máximo de correlações em relação ao grupo de materiais testados.

\section{RESULTADOS E DISCUSSÃO}

Um dos principais objetivos em programas de melhoramento é a obtenção de cultivares mais produtivas. Contudo, outras características devem também ser melhoradas conjuntamente, ressaltando a importância de se conhecer o comportamento associativo entre os vários caracteres de interesse, pois a correlação existente pode vir a dificultar ou a facilitar o processo de seleção de caracteres em associação.

Pelo resumo da análise de variância conjunta apresentado na Tabela 1, pode-se observar que ocorreram interações significativas dos genótipos com os ambientes apenas quanto ao caráter produtividade de grãos (PG), os quais apresentaram valores de F menores do que os obtidos em relação aos tratamentos. Com isso, pode-se supor que não ocorreu interação do tipo complexa segundo Gomez \& Gomez (1983), e as estimativas de correlações puderam ser obtidas a partir da análise de variância conjunta sem restrições.

Na Tabela 2 são apresentadas as estimativas de correlações obtidas em plantas individuais e médias de parcelas. As correlações baseadas em parcelas (Johnson et al., 1955) e em médias de progênies (Bravo et al., 1980), geralmente são mais consistentes em relação àquelas obtidas com base em plantas individuais.

Resultados de interesse para um programa de melhoramento de soja que vise a obtenção de materiais promissores do tipo alimento podem ser observados na Tabela 2. As correlações entre número de dias para maturidade (NDM) e peso de cem sementes (PCS) nos topocruzamentos de sementes grandes apresentaram valores negativos $(-0,523 \mathrm{e}$ $-0,391)$, o que significa tendência de plantas com sementes maiores serem mais precoces. Nos topocruzamentos envolvendo materiais de sementes pequenas, ao contrário, a correlação apresentou valor positivo $(0,408)$, ou seja, quanto menor o tamanho das sementes mais precoces foram as plantas, o que é interessante para esta categoria (sementes pequenas). Por serem categorias extremas, e a seleção é direcionada para os extremos, os valores de correlações desses dois caracteres indicam a possibilidade de facilitar o processo de seleção. Conforme Pacova (1992), esse comportamento inverso dos grupos de sementes grandes em relação ao de sementes pequenas pode ser conseqüência de limitações fisiológicas inerentes, necessitando-se a adoção de estratégias de seleção diferentes para cada grupo.

Entre valor agronômico (VA) e peso de cem sementes (PCS) (Tabela 2), observa-se que, nos topocruzamentos de sementes grandes, tanto com Doko como com FT-2 as correlações apresentaram valores negativos $(-0,629$ e $-0,685)$; ou seja, as sementes maiores estavam associadas às piores notas de VA. Uma explicação para tal fato decorre de que plantas de sementes grandes produzem menor número de vagens, com isso, o aspecto da planta, quanto à produtividade, fica prejudicado e a nota tende a diminuir. Comportamento distinto pôde ser notado nos topocruzamentos envolvendo materiais de sementes pequenas, em que o menor tamanho do grão esteve associado a notas inferiores (correlação 0,737). Este fato é explicado a campo pelo menor tamanho das 
TABELA 1. Resumo da análise de variância conjunta dos caracteres número de dias para maturidade (NDM), valor agronômico (VA), peso de 100 sementes (PCS), produtividade de grãos (PG) e largura visual das vagens (LVV) em topocruzamentos de soja com sementes grandes (Gg) e com sementes pequenas $(\mathrm{Gp})$.

\begin{tabular}{lccccc}
\hline Fonte de variação & \multicolumn{5}{c}{ Quadrado médio } \\
\cline { 2 - 6 } & NDM & VA & PCS & PG & LVV \\
\hline Ambientes (E) & $843,9^{* *}$ & $4,68^{* *}$ & $17,79^{*}$ & $1085,6^{* *}$ & $3,48^{* *}$ \\
Tratamentos (G) & $433,0^{* *}$ & $1,09^{* *}$ & $102,26^{* *}$ & $892,5^{* *}$ & $1,61^{* *}$ \\
Sem. grandes (Gg) & $360,9^{* *}$ & $1,06^{* *}$ & $54,94^{* *}$ & $886,7^{* *}$ & $1,05^{* *}$ \\
Sem. pequenas (Gp) & $480,3^{* *}$ & $0,32^{* *}$ & 5,97 & 385,5 & 0,21 \\
G x E & 41,1 & 0,09 & 4,02 & $378,7^{* *}$ & 0,13 \\
Gg x E & 42,9 & 0,09 & 4,25 & $433,9^{* *}$ & 0,13 \\
Gp x E & 29,5 & 0,06 & 1,27 & 74,0 & 0,07 \\
Resíduo & 34,4 & 0,09 & 4,57 & 224,5 & 0,11 \\
\hline
\end{tabular}

*e $* *$ Significativo a $5 \%$ e $1 \%$ de probabilidade, respectivamente.

TABELA 2. Estimativas de correlações fenotípicas para os caracteres peso de cem sementes (PCS), número de dias para maturidade (NDM), valor agronômico (VA), produtividade de grãos (PG) e largura visual das vagens (LVV), em médias de parcelas e de plantas individuais (entre parênteses), para topocruzamentos envolvendo parentais exóticos de sementes grandes e pequenas com Doko e FT-2 .

\begin{tabular}{|c|c|c|c|}
\hline Caracteres & $\begin{array}{c}\text { Sem. grandes } \\
\mathrm{x} \\
\text { Doko }\end{array}$ & $\begin{array}{c}\text { Sem. grandes } \\
\text { x } \\
\text { FT-2 }\end{array}$ & $\begin{array}{c}\text { Sem. pequenas } \\
\mathrm{x} \\
\text { (Doko e FT-2) }\end{array}$ \\
\hline NDM x PCS & $\begin{array}{c}-0,523 * * \\
(-0,142 * *)\end{array}$ & $\begin{array}{l}-0,391^{*} \\
\left(0,013^{\text {ns }}\right)\end{array}$ & $\begin{array}{c}0,408^{\text {ns }} \\
\left(-0,031^{\text {ns }}\right)\end{array}$ \\
\hline VA $x$ PCS & $\begin{array}{c}-0,629 * * \\
(-0,159 * *)\end{array}$ & $\begin{array}{c}-0,685^{* *} \\
\left(-0,202^{* *}\right)\end{array}$ & $\begin{array}{c}0,737^{\mathrm{ns}} \\
\left(0,106^{\mathrm{ns}}\right)\end{array}$ \\
\hline $\mathrm{PG} \times \mathrm{PCS}$ & $\begin{array}{c}-0,490 * * \\
(-0,064 * *)\end{array}$ & $\begin{array}{c}-0,644^{* *} \\
\left(-0,088^{* *}\right)\end{array}$ & $\begin{array}{c}0,500^{\mathrm{ns}} \\
\left(0,127^{*}\right)\end{array}$ \\
\hline LVV x PCS & $\begin{array}{c}0,895^{* *} \\
(0,522 * *)\end{array}$ & $\begin{array}{c}0,912 * * \\
(0,480 * *)\end{array}$ & $\begin{array}{c}0,742^{\mathrm{ns}} \\
\left(0,528^{* *}\right)\end{array}$ \\
\hline
\end{tabular}

ns,$*$ e ** Não-significativo e significativo a $5 \%$ e $1 \%$ de probabilidade, respectivamente.

vagens apresentar aspecto visual pior, levando também à diminuição da nota de VA. Ambas as constatações caracterizam dificuldades para uma seleção simultânea dos dois caracteres. Mas com sementes pequenas a dificuldade é menos limitante, pois materiais no limite superior da categoria broto/"natto" apresentaram visual adequado. Portanto, a seleção de plantas com sementes maiores, interessante à cate- goria de soja hortaliça, com a tendência de redução na nota de VA, pode dificultar o melhoramento simultâneo de ambos os caracteres.

Para a correlação PG com PCS (Tabela 2), os topocruzamentos entre os parentais exóticos de sementes grandes e FT-2 apresentaram problemas, pois os valores estimados entre os dois caracteres (correlação de -0,644) e também para aqueles envolvendo o parental Doko (correlação de -0,490) foram negativos, recomendando-se uma seleção cuidadosa para não prejudicar o tamanho das sementes nem a produção. Valores similares foram obtidos por Rao \& Sharma (1985), -0,54 entre PCS e produção de sementes/planta, e Kaw \& Menon (1972), com -0,44. Resultados contrastantes foram observados por Konovsky et al. (1996) estudando 36 cultivares de soja hortaliça, cuja correlação de peso de sementes e produção bruta foi de 0,57, e por Saka et al. (1996), com valor de 0,43 entre PG e PCS, estes valores contrastantes podem ter ocorrido em virtude dos diferentes genótipos , o tipo e tamanho de parcela e as próprias diferenças ambientais. Nos topocruzamentos de sementes pequenas, os menores tamanhos de sementes associaram-se a menores produções (correlação de 0,500). Assim, deve-se ter cuidado num processo de seleção envolvendo os dois caracteres, tanto para os topocruzamentos de sementes grandes como para os de sementes pequenas.

A largura visual de vagem (LVV) é vista como um caráter potencial para agilizar a seleção, sendo extremamente fácil de ser avaliada. Desse modo, a 
avaliação da correlação entre este caráter e o peso de cem sementes é de suma importância, dada a possibilidade de se substituir o PCS pela LVV, aumentando a eficácia do processo de seleção. Os topocruzamentos de sementes grandes e os de sementes pequenas apresentaram estimativas de correlação (Tabela 2) que, sem dúvida, indicam que LVV pode ser usado em substituição a PCS, ou seja, correlações sempre elevadas e positivas $(0,895,0,912$ e 0,742 ). Os valores aproximaram-se daqueles obtidos por Saka et al. (1996), cuja correlação observada foi de 0,96 . Os resultados concordam também com os de Bravo et al. (1980), Fraser et al. (1982), Cianzio et al. (1982) e Shanmugasundaram \& Chung-Ruey (1982).

De maneira geral, as estimativas de correlações em plantas individuais, confirmam aquelas obtidas em médias de parcelas; senão tanto em magnitude pelo menos em direção (sinal da correlação), excetuando-se a correlação de NDM com PCS para o grupo dos topocruzamentos de sementes grandes com FT-2 e para o de sementes pequenas (Tabela 2).

As estimativas de correlações fenotípicas baseadas na média de todos os tratamentos é o procedimento mais encontrado na literatura, o que impossibilita um estudo do comportamento específico de cada tratamento, pelas diferenças genéticas em interação com o ambiente. A obtenção de correlações mínimas e máximas para cada topocruzamento e o minucioso estudo desses valores, permite que se visualize as diferenças existentes entre eles. Nos topocruzamentos de sementes grandes (Tabela 3), tanto com Doko como com FT-2, as correlações com PCS variaram de médias negativas a médias positivas para
NDM, VA e PG; semelhante ao observado por Johnson et al. (1955), Burris et al. (1973), Harrison et al. (1981) e Santos (1988). Esses autores obtiveram valores de correlação genotípica entre PCS e PG variando de $-0,59$ a 0,66 . Isso significa uma dificuldade em se compreender realmente o que aconteceria se fosse selecionado um caráter em detrimento do outro.

Entre LVV e PCS, dentro dos topocruzamentos de sementes grandes (Tabela 3), houve uma tendência de as correlações variarem de baixas a médias positivas $(-0,127$ a 0,705$)$. O resultado reforça a conclusão anterior de que talvez seja possível aumentar o tamanho de sementes selecionando-se vagens mais largas.

Dentro dos topocruzamentos de sementes pequenas (Tabela 3), as correlações apresentaram comportamento distinto em relação aos de sementes grandes. Entre NDM e PCS as estimativas apresentaram somente valores negativos baixos nos topocruzamentos com Doko $(-0,336$ a $-0,185)$, e praticamente nulos naqueles com FT-2 (-0,066 a 0,087). Entre VA e PCS, dentro dos topocruzamentos com Doko, as correlações apresentaram valores negativos a positivos $(-0,157$ a 0,307$)$, relativamente baixos, devendo então atentar-se para a seleção dentro de topocruzamentos. Já entre os topocruzamentos com FT-2 ocorreram apenas valores baixos de correlação entre esses caracteres $(0,074$ a 0,099$)$. Entre PG e PCS, nos topocruzamentos com Doko as correlações foram sempre próximas de zero $(-0,080$ a 0,089$)$; já naqueles com FT-2, ocorreram correlações positivas baixas a médias $(0,166$ a 0,429$)$. Neste caso evidencia-se uma certa dificuldade para

TABELA 3. Valores máximos e mínimos das estimativas de correlações fenotípicas de Pearson obtidos dentro de cada topocruzamento de sementes grandes e de sementes pequenas com Doko e com FT-2.

\begin{tabular}{|c|c|c|c|c|c|c|c|c|}
\hline \multirow[t]{2}{*}{ Caracteres $^{1}$} & \multicolumn{2}{|c|}{$\begin{array}{c}\text { Sem. grandes } \\
\text { x } \\
\text { Doko } \\
\end{array}$} & \multicolumn{2}{|c|}{$\begin{array}{c}\text { Sem. grandes } \\
\text { x } \\
\text { FT-2 } \\
\end{array}$} & \multicolumn{2}{|c|}{$\begin{array}{c}\text { Sem. pequenas } \\
\text { x } \\
\text { Doko } \\
\end{array}$} & \multicolumn{2}{|c|}{$\begin{array}{c}\text { Sem. pequenas } \\
\text { x } \\
\text { FT-2 } \\
\end{array}$} \\
\hline & Mín. & Máx. & Mín. & Máx. & Mín. & Máx. & Mín. & Máx. \\
\hline NDM $x$ PCS & $-0,489 * *$ & $0,534 * *$ & $-0,342^{\text {ns }}$ & $0,476^{* *}$ & $-0,336^{* *}$ & $-0,185^{\mathrm{ns}}$ & $-0,066^{\mathrm{ns}}$ & $0,087^{\mathrm{ns}}$ \\
\hline VA $x$ PCS & $-0,407^{*}$ & $0,364 * *$ & $-0,491 * *$ & $0,319^{\mathrm{ns}}$ & $-0,157^{\mathrm{ns}}$ & $0,307 *$ & $0,074^{\mathrm{ns}}$ & $0,099^{\mathrm{ns}}$ \\
\hline PG $\times$ PCS & $-0,380 * *$ & $0,399 * *$ & $-0,482 * *$ & $0,326^{* *} *$ & $-0,080^{\mathrm{ns}}$ & $0,089^{\mathrm{ns}}$ & $0,166^{\mathrm{ns}}$ & $0,429 * *$ \\
\hline LVV x PCS & $-0,008^{\mathrm{ns}}$ & $0,705 * *$ & $-0,127^{\mathrm{ns}}$ & $0,558 * *$ & $0,428 * *$ & $0,575^{* *}$ & $0,299 *$ & $0,672 * *$ \\
\hline
\end{tabular}

${ }^{1}$ PCS: peso de cem sementes; NDM: número de dias para maturidade; VA: valor agronômico; PG: produtividade de grãos; LVV: largura visual das vagens. ns, * e ** Não-significativo e significativo a $5 \%$ e $1 \%$ de probabilidade, respectivamente. 
a seleção de plantas mais produtivas com sementes menores, sendo os menores PCS interessantes para essa categoria. Entre LVV e PCS em sementes pequenas (Tabela 3) foram observados valores positivos baixos a médios $(0,299$ a 0,672$)$, indicando a facilidade de seleção de um caráter com base no outro, podendo-se substituir o processo de obtenção de PCS, que é extremamente trabalhoso, pela LVV, de rápida e fácil aplicabilidade.

Pacova (1992) verificou que os valores de correlação são diferentes com o tipo (grão, broto/"natto" e hortaliça) de soja envolvido. O mesmo foi observado no presente trabalho, no qual o comportamento associativo dos caracteres variou consideravelmente, conforme os parentais envolvidos nos topocruzamentos, caso fossem com sementes grandes ou pequenas.

Considerando a grande variação nos resultados dentro de topocruzamentos e entre eles, recomendase que estimativas de correlação obtidas com base em médias de parcelas sejam aferidas dentro dos cruzamentos, para uma avaliação mais precisa da possibilidade de se selecionar um caráter, indiretamente, por meio de outro. Convém ressaltar que os valores estimados de correlação fenotípica referem-se ao universo dos topocruzamentos envolvidos na pesquisa, restringindo-se sua interpretação e utilização para fins de seleção apenas nestes materiais.

\section{CONCLUSÕES}

1. O caráter largura visual das vagens substitui perfeitamente o peso de cem sementes, com base nas correlações estimadas.

2. Estratégias diferentes de seleção são mais apropriadas para cada grupo de topocruzamento (sementes grandes e sementes pequenas), em virtude das diferentes estimativas de correlações.

3. O caráter valor agronômico apresenta correlações fenotípicas indesejáveis com peso de cem sementes, dificultando a seleção baseada em ambos os caracteres.

4. As estimativas de correlação fenotípica para cada topocruzamento apresentam as diferenças de comportamento, inerente aos genótipos testados.

\section{AGRADECIMENTOS}

À FAPESP, pelo auxílio concedido para a pesquisa; a Vera Maria Quecini, pela correção do abstract.

\section{REFERÊNCIAS}

BRAVO, J.A.; FEHR, W.R.; CIANZIO, S.R. de. Use of pod width for indirect selection of seed weight in soybeans. Crop Science, Madison, v.20, p.507-510, 1980.

BURRIS, J.S.; EDJE, O.T.; WAHAB, A.H. Effects of seed size on seedling performance in soybeans. II. Seedling growth and photosynthesis and field performance. Crop Science, Madison, v.13, p.207-210, 1973.

CANTO, W.L.; TURATTI, J.M. Produção e mercado de produtos intermediários protéicos de soja no Brasil. Boletim do Centro de Pesquisa de Processamento de Alimentos, Curitiba, v.7, n.2, p.111-139, 1989.

CIANZIO, S.R. de; FRANK, S.J.; FEHR, W.R. Seed width to pod width ratio for identification of green soybean pods that have attained maximum length and width. Crop Science, Madison, v.22, p.462-466, 1982.

FEHR, W.R.; CAVINESS, C.E. Stages of soybean development. Ames : Iowa State University of Science and Technology, 1977. 12p. (Special Report, 80).

FRASER, J.; EGLI, D.B.; LEGGETT, J.E. Pod and seed development in soybeans cultivars with differences in seed size. Agronomy Journal, Madison, v.74, p.81-85, 1982.

GOMEZ, K.A.; GOMEZ, A.A. Regression and correlation analysis. In: GOMEZ K.A.; GOMEZ, A.A. (Ed.). Statistical procedures for agricultural research. New York: J. Wiley, 1983. p.357-423.

HARRISON, S.A.; BOERMA, H.R.; ASHLEY, D.A. Heritability of canopy-apparent photosynthesis and its relationship to seed yield in soybeans. Crop Science, Madison, v.21, p.222-226, 1981.

JOHNSON, H.W.; ROBINSON, H.F.; COMSTOCK, R.E. Genotypic and phenotypic correlations in soybeans and their implications in selection. Agronomy Journal, Madison, v.47, n.10, p.477-483, 1955.

KAW, R.N.; MENON, P.M. Association between yield and components in soybean. Indian Journal of Genetics 
and Plant Breeding, Calcutta, v.32, p.276-280, 1972.

KONOVSKY, J.; EVANS, D.W.; LUMPKIN, T.A. Habitability of yield, plant architecture, and quality traits of Edamame: the vegetable soybean. Soybean Genetics Newsletter, Ames, v.23, p.243-249, 1996.

MORO, G.L.; REIS, M.S.; SEDIYAMA, C.S.; SEDIYAMA, T.; OLIVEIRA, A.B. Correlações entre alguns caracteres agronômicos em soja (Glycine $\max ($ L.) Merrill). Revista Ceres, Viçosa, v.39, n.223, p.225-232, 1992.

PACOVA, B.E.V. Análise genética de progênies segregantes de soja apropriada para o consumo humano. Piracicaba : ESALQ, 1992. 217p. Tese de Doutorado.

RAO, S.K.; SHARMA, S.M. Genetic variation for pod characteristics and their inter-relationships with field germinability in soybean. Indian Journal of Agricultural Research, Karnal, v.19, n.3, p.150-154, 1985.
SAKA, K.; SHIPE, E.R.; WALLACE, S.U. Relationships among plant characters in soybean with different seed size. Soybean Genetics Newsletter, Ames, v.23, p.225-229, 1996.

SANTOS, A.L.C. Variabilidade entre genótipos de soja (Glycine $\max (\mathrm{L}$.) Merrill) nas fases imatura e madura de desenvolvimento. Piracicaba : ESALQ, 1988. 135p. Dissertação de Mestrado.

SHANMUGASUNDARAM, S.; CHUNG-RUEY, Y. Screening for immature green soybeans as a vegetable. Soybean Genetics Newsletter, Ames, v.9, p.95-96, 1982.

VELLO, N.A. Ampliação da base genética do germoplasma e melhoramento da soja na ESALQUSP. In: SIMPÓSIO SOBRE CULTURA E PRODUTIVIDADE DA SOJA, 1., 1991, Piracicaba. Anais. Piracicaba : FEALQ, 1992. p.60-81.

VENCOVSKY, R. Associação entre caracteres. In: VENCOVSKY, R.; BARRIGA, P. (Ed.). Genética biométrica no fitomelhoramento. Ribeirão Preto : Sociedade Brasileira de Genética, 1992. p.335-434. 